\title{
Force Monitor for Training Manual Skills in the Training of Chiropractors
}

\author{
Juan-Mario Gruber ${ }^{1}$, Daniel Mühlemann ${ }^{2}$, Darius Eckhardt ${ }^{1}$ and Ibrahim Evren ${ }^{1}$ \\ ${ }^{1}$ Institute of Embedded Systems Zurich University of Applied Sciences, Winterthur, Switzerland \\ ${ }^{2}$ Department of Chiropractic Medicine, University of Zurich, Switzerland
}

Keywords: $\quad$ Spinal Manipulation, Training, Skill, Biomechanics, Teaching Aid, Force Monitoring Device, Biofeedback.

\begin{abstract}
As part of their training, students of Chiropractic Medicine at Zürich are trained to acquire and then improve their manual and manipulative skills, especially their ability to deliver manipulative thrusts with a defined preloading force, an impulse that is delivered with an adequate and reproducible force within a defined time without letting up on the preload-pressure. In order to facilitate this process, objective feedback is paramount. This led to the idea of developing a force-measurement and -monitoring system. The newly developed system consists of a wireless device with a force sensor and an app that is running on standard smartphones. The device records the force applied to the sensor and transmits it via Bluetooth Low Energy (BLE) to the app. There it is visualised as a graph and can be evaluated. The system allows us to provide all students with a tool to develop their manual skills, and especially their thrusting technique. As the feedback given by the system can be recorded, progress can be monitored and students can be mentored accurately according to their strengths and weaknesses.
\end{abstract}

\section{INTRODUCTION}

Chiropractic Medicine at Zürich University is a 6year program consisting of a 3-year Bachelor's degree in Medicine and a 3-year Master's degree in Chiropractic Medicine at the university's medical faculty. Throughout the curriculum, students are instructed and trained to acquire and then constantly improve their manual and manipulative skills.

To support the students training it is necessary for the instructors to be able to give objective feedback.

\section{FAST, ACURATE AND REPRODUCABLE MANUAL THRUSTS}

One particular challenge has been to train the student's ability to deliver manipulative thrusts with a defined preloading force, an impulse that is delivered with an adequate and reproducible force within a time of 100-150 ms without letting up on the preload-pressure.

\subsection{Typical Force Path}

A graph showing the correct delivery of a high velocity, low amplitude thrust is shown in figure 1 .

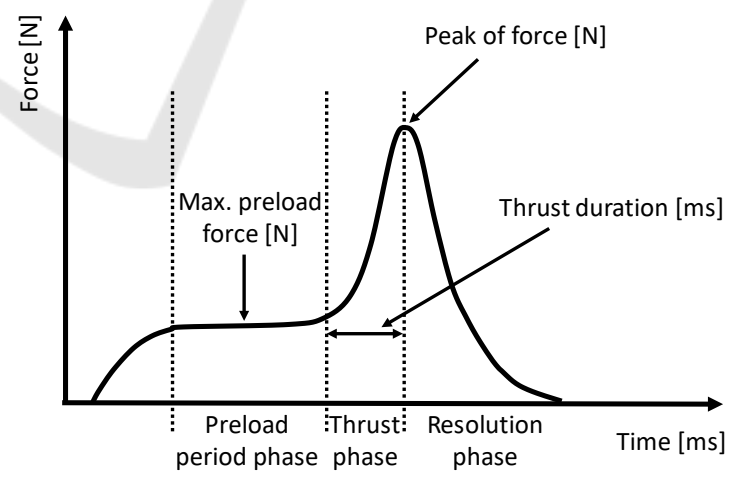

Figure 1: Correct delivery of a high velocity, low amplitude thrust (Triano et al., 2004; Triano et al., 2002; Triano et al., 2012).

The challenge is to deliver a reproducible preloadforce, which may vary with the technique, then a fast, accurate, and reproducible thrust (impulse), which may vary again according to the technique chosen and then to back off completely as fast as possible (unload). The slew rate in both delivering 
the impulse as well as in unloading must be as high as possible, the impulse as a whole as short as possible. A typical mistake is to reduce the preload prior to thrust-delivery (figure 2, upper graph).
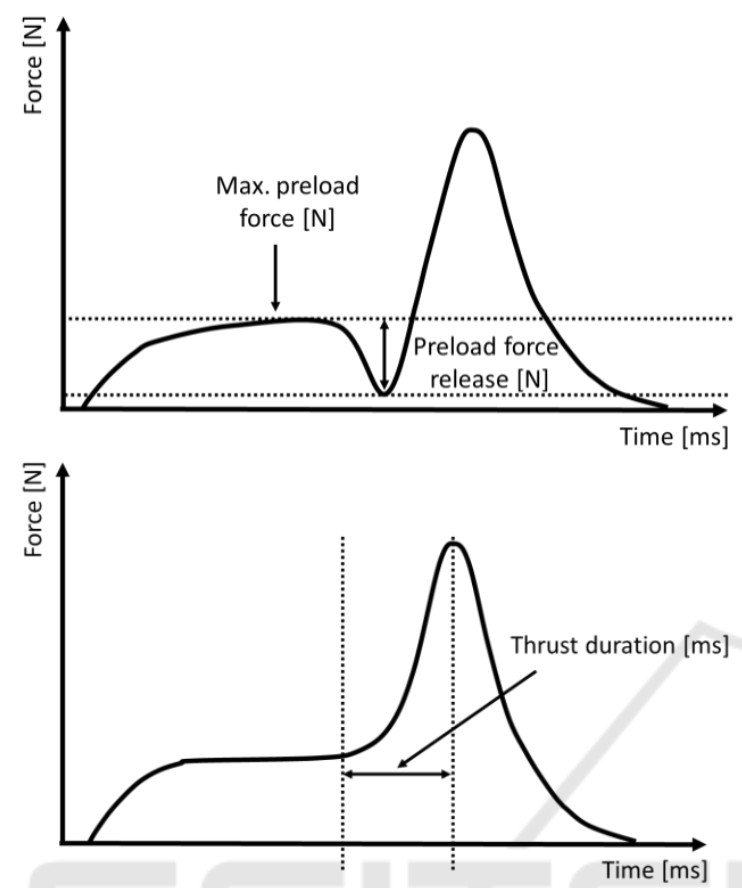

Figure 2: Incorrect delivery (upper graph) against correct delivery (lower graph).

\subsection{Using Force Measurement Systems in Chiropractic Education}

In the past years, force sensors have been developed to visualize the impulses delivered.

The students are able to analyse and compare the force path during practice. To improve mobility and handling, the latest version works with a force sensor connected via Bluetooth to a smartphone to visualize the data.

This newly developed system allows us to provide all students with a tool to develop their manual skills, and especially their thrusting technique. As the feedback given by the system can be recorded, progress can be monitored and students can be mentored accurately according to their strengths and weaknesses.

\section{FORCE MONITORING DEVICE}

The developed system consists of a wireless device with a force sensor and an app that is running on standard smartphones. Figure 3 is showing the prototype.

The device measures an applied force and transmits it via Bluetooth Low Energy (BLE) to the smartphone. The app evaluates the data and displays it as a graph.

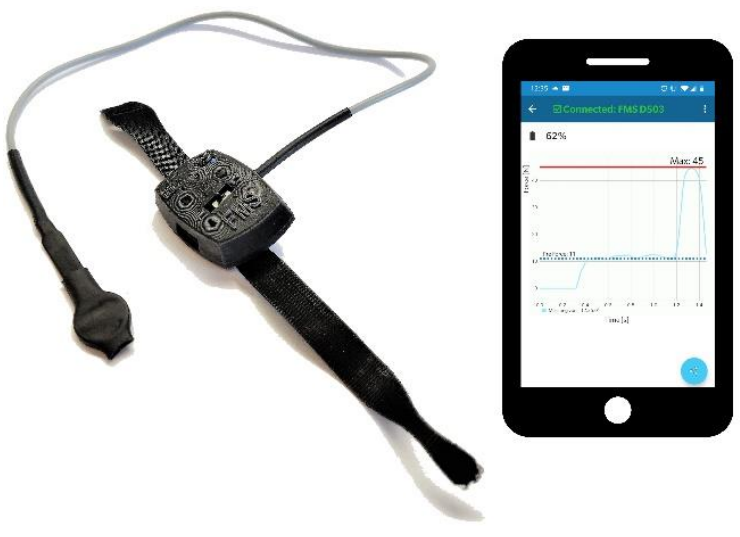

Figure 3: Prototype.

\subsection{System Concept}

The System consists of two main parts: The embedded electronics with sensor and the smartphone application (Figure 4).

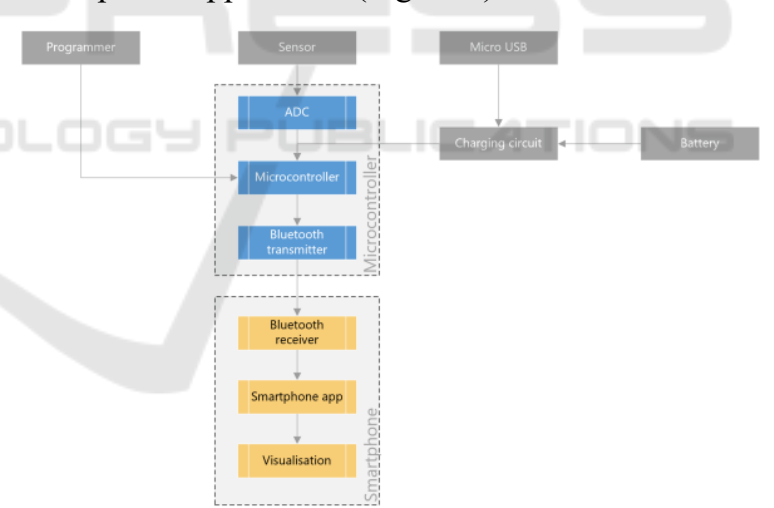

Figure 4: System Block Diagram.

The sensor measures the applied force. The microcontroller device reads the analogue data, converts it into digital data and transmits it via Bluetooth Low Energy to the smartphone. An Android app receives the data, does calculations and visualises it as a graph. Further data analysis is made to show a preload-force and the maximum force. In addition, the data can be exported and shared. The device and the app are simple to use. 


\subsection{Sensor}

The sensor is built of a force sensitive resistor (FSR) in combination with two metal plates. It changes the resistance when a force is applied. The sensor is quite affordable, but it is nonlinear. This is why it needs additional compensation during calibration. During calibration an accurate piezoelectric sensor is used as reference and mounted into a hand press. Several reference points are measured over a range of 500 Newton and interpolated for the FSR. This results in a reasonable accuracy of the FSR (Figure $5)$.

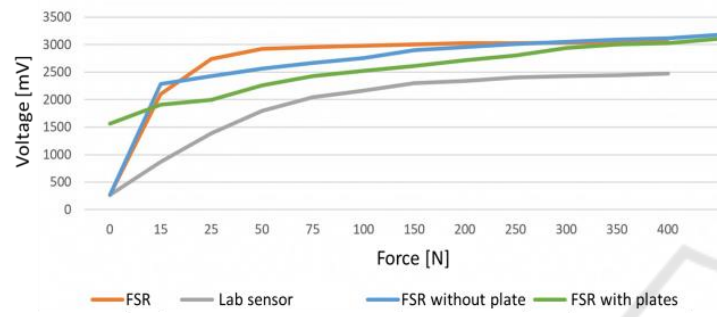

Figure 5: Sensor calibration of different sensor types.

\subsection{Measurement Device}

The measure device is in a custom-built case. It is worn at the wrist of the user.

For the prototype this case is produced with a 3D printer based on the stereolithographic (SLA) principle. The electronics are integrated on a custom printed circuit board (PCB). A system on chip (SoC) from Nordic Integrated, the $n R F 52840$ model, is the core of the device. This is a Microcontroller with a built in Bluetooth module. The device is powered by a rechargeable lithium battery. It can be charged by a micro USB connector. The sensor is attached to a short cable, which allows the user to measure a force at different positions, such as at the heel of the hand.

By sliding the main switch, the device is powered on and is visible over BLE. When connected to the app, the device starts to read the sensor data (Figure 6).

As soon as a force is applied to the force sensor, the device is recording the data. When the sensor is released, the raw sensor data is converted into a force and transmitted over BLE to the app.

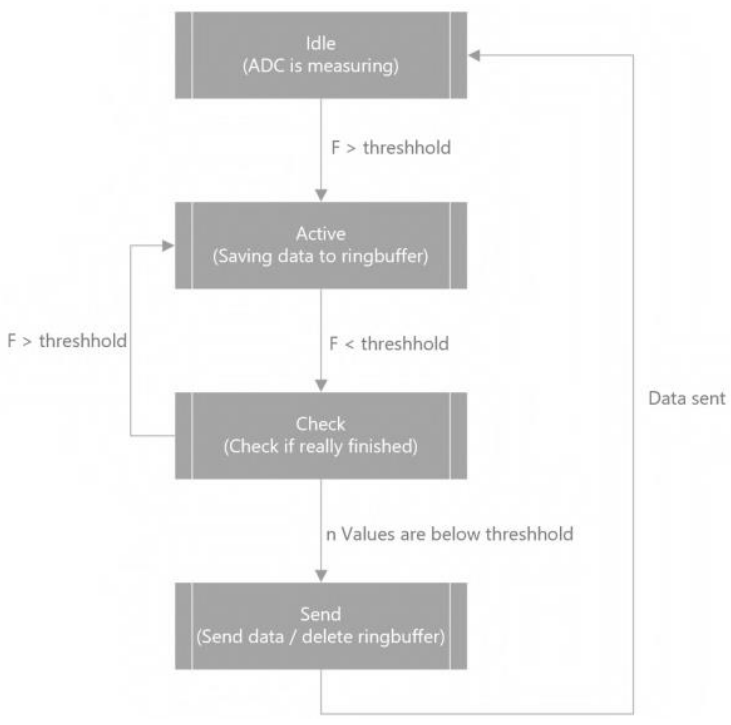

Figure 6: State Machine Measurement.

\subsection{Smartphone Application}

An Android App has been developed as a userfriendly interface. The aim of the App is to visualize the applied Force and highlight for chiropractor students useful data such as the peak force and the preload force of an exercise (Figure 7).

When a measurement is started a starting flag is sent to display this in the app. At the end of a measurement the data is transmitted to the app.

The data evaluation starts as soon as the required measurement data has been received. The starter packet passes a byte with the value 0x6 and lets the display know that the measurement has started. Before visualization, the received bytes are checked for completeness and then buffered in a 2dimensional array. For the representation an array list with the respective $\mathrm{x}$ - and $\mathrm{y}$-coordinates is created. The $\mathrm{x}$-axis is defined by the scanning frequency and can be returned for the respective $y$ values. The graph is plotted and the maximum force as well as the pre-force is shown as numbers. This helps the chiropractic students to assess whether the impulse was produced directly after the pre-pressure or whether the pre-pressure has decreased. Furthermore an export function has been added to save the measured force locally on the phone and to share the .csv File via installed Apps on the Smartphone. 


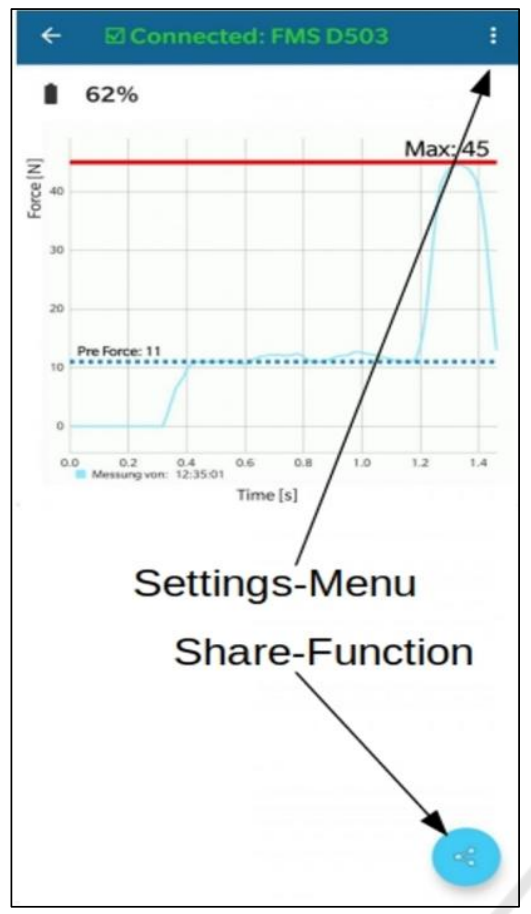

Figure 7: App showing force diagram.

\subsection{Wireless Communication}

The Connection between the Device and the Smartphone is established via Bluetooth Low Energy, while the Smartphone acts as the BLECentral and the device as the BLE-Peripheral. The Connection can be established in three steps assuming the device is switched 'On'. The device starts in Advertising-Mode automatically. The app scans at start for nearby compatible devices which are advertising and will show a list of all available FMS-devices. After choosing a device a connection using BLE GATT will be established and the system is ready for data transfer of measured values.

\section{DEVICE TESTS AND CONCLUSIONS}

\subsection{Test Setup}

The device was tested for accuracy and consistency with an accurate piezoelectric sensor and a hand press. Furthermore, the battery duration was tested. Finally, the device was tested by chiropractor students. Primarily the user friendliness and the utility of the system were tested.

\subsection{Lab Tests}

During the accuracy test of the force value the medium deviancy from the actual value was $7 \%$ (Table 1).

Table 1: Sensor Accuracy.

\begin{tabular}{|c|c|c|c|c|c|c|c|}
\hline Accuracy & Maxdev & & $10 \%$ & & & & \\
\hline Setpoint in N & 25 & 50 & 75 & 100 & 200 & 300 & \\
\hline Actual value in $\mathrm{N}$ & 27 & 52 & 80 & 107 & 218 & 285 & 371 \\
\hline Deviation in $\mathrm{N}$ & 2 & 2 & 5 & 7 & 18 & 15 & 29 \\
\hline Deviation & $8 \%$ & $4 \%$ & $7 \%$ & $7 \%$ & $9 \%$ & $5 \%$ & $7 \%$ \\
\hline
\end{tabular}

This is in the acceptable range, because the relative value is more important than the absolute. In the consistency test the mean deviation was 9 Newton. The battery test identified a system uptime of almost 50 hours. This means that the device needs to be recharged after 24 days if it is used for two hours per day.

\subsection{Practical Tests}

At Zürich University instrumented bio-feedback has been used for teaching motor skills for a few years with a predecessor device.

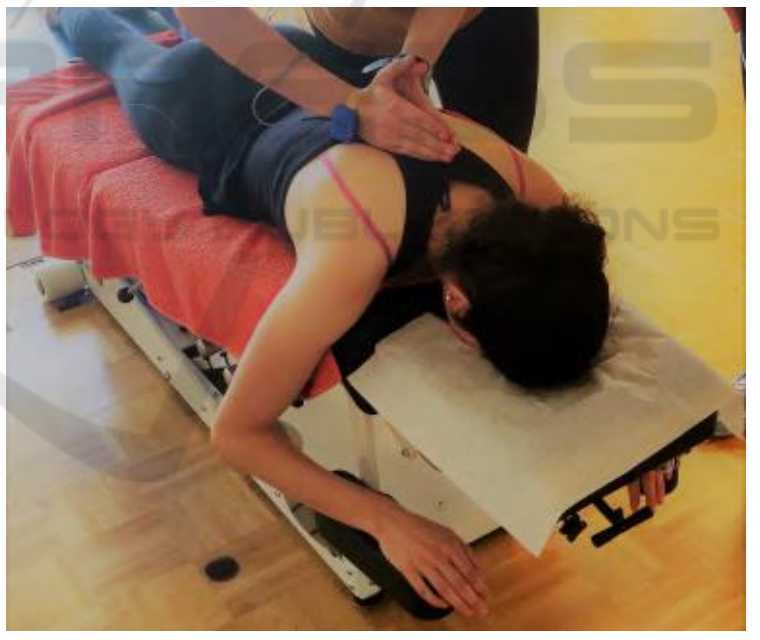

Figure 8: Device Test at University Zurich.

The results regarding the improvement of motorand manipulative skills of the students are very good. The students appreciate the immediate feedback on their performance and are motivated to take the initiative to improve on their manual skillsdeficits.

The new model is easier to use, as it is highly portable and, using a smartphone interface, PCindependent which will make it very attractive for the students. The feedback from our students of 
Chiropractic Medicine was mostly positive (Figure 9).

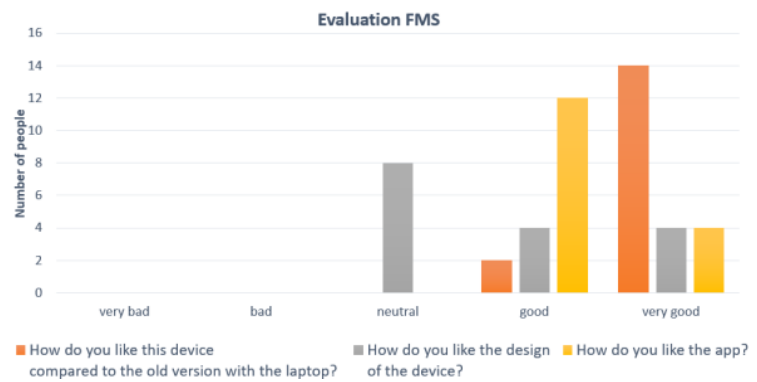

Figure 9: Feedback from Acceptance Test.

$88 \%$ liked the device very much, the others liked it. No one had problems connecting the device to the app. $81 \%$ would appreciate it if there was as well an iOS app for the system.

\section{REFERENCES}

Triano Jj1, Bougie J, Rogers C, Scaringe J, Sorrels K, Skogsbergh D, Mior S., 2004. Procedural skills in spinal manipulation: do prerequisites matter? In The Spine Journal. Sep-Oct;4(5):557-63.

Triano JJ1, Rogers CM, Combs S, Potts D, Sorrels K., 2002. Developing skilled performance of lumbar spine manipulation. In Journal of manipulative and physiological therapeutics. Jul-Aug;25(6):353-61.

Triano Jj1, Descarreaux M, Dugas C., 2012. Biomechanics--review of approaches for performance training in spinal manipulation. In Journal of electromyography and kinesiology. Oct;22(5):732-9. 